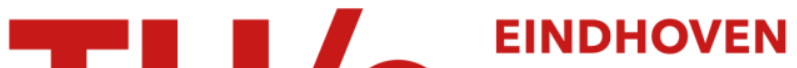 UNIVERSITY OF TECHNOLOGY
}

\section{An MPEG decoder with embedded compression for memory reduction}

Citation for published version (APA):

With, de, P. H. N., Frencken, P. H., \& Schaar, van der, M. (1998). An MPEG decoder with embedded compression for memory reduction. IEEE Transactions on Consumer Electronics, 44(3), 545-555.

https://doi.org/10.1109/30.713162

DOI:

$10.1109 / 30.713162$

Document status and date:

Published: 01/01/1998

\section{Document Version:}

Publisher's PDF, also known as Version of Record (includes final page, issue and volume numbers)

\section{Please check the document version of this publication:}

- A submitted manuscript is the version of the article upon submission and before peer-review. There can be important differences between the submitted version and the official published version of record. People interested in the research are advised to contact the author for the final version of the publication, or visit the $\mathrm{DOI}$ to the publisher's website.

- The final author version and the galley proof are versions of the publication after peer review.

- The final published version features the final layout of the paper including the volume, issue and page numbers.

Link to publication

\section{General rights}

Copyright and moral rights for the publications made accessible in the public portal are retained by the authors and/or other copyright owners and it is a condition of accessing publications that users recognise and abide by the legal requirements associated with these rights.

- Users may download and print one copy of any publication from the public portal for the purpose of private study or research.

- You may not further distribute the material or use it for any profit-making activity or commercial gain

- You may freely distribute the URL identifying the publication in the public portal.

If the publication is distributed under the terms of Article 25fa of the Dutch Copyright Act, indicated by the "Taverne" license above, please follow below link for the End User Agreement:

www.tue.nl/taverne

Take down policy

If you believe that this document breaches copyright please contact us at:

openaccess@tue.nl

providing details and we will investigate your claim. 


\title{
AN MPEG DECODER WITH EMBEDDED COMPRESSION FOR MEMORY REDUCTION
}

\author{
Peter H.N. de With ${ }^{1}$, Peter H. Frencken ${ }^{2}$ and Mihaela v.d. Schaar-Mitrea ${ }^{3}$ \\ ${ }^{1}$ University of Mannheim, Dept. Circuitry \& Simulation, B6-26, 68131 Mannheim, Germany \\ ${ }^{2}$ Philips Semiconductors, Systems Laboratory Eindhoven, Hurksestraat, Eindhoven, The Netherlands \\ ${ }^{3}$ Philips Research Labs., Prof. Holstlaan 4, 5656 AA Eindhoven, The Netherlands
}

\begin{abstract}
We present an MPEG decoder with reduced system costs by employing embedded compression of the reference frames which are used for motioncompensated (MC) decoding. The embedded compression scheme used is based on either blockpredictive coding or DCT transform coding, depending on the required memory compression. The compression features simple recovery of $(M C)$ block data, while preventing visible artifacts. The compression was optimized for low costs, enabling real application in a commercial MPEG IC. It was found that the same techniques can be applied in the encoder as well.
\end{abstract}

\section{Introduction}

The MPEG video compression standard [1] is being applied in numerous applications, such as video communication in the multimedia $\mathrm{PC}$, Digital Video Broadcasting (DVB) and more recently, storage of video sequences on the Digital Versatile Disk (DVD). The evolution of the aforementioned applications is clearly towards large-scale usage in the consumer electronics market, where low system costs are of utmost importance and a continuous price erosion occurs. In this paper, a new technical solution is proposed to realize lower system costs for an MPEG decoder, without any significant loss in the resulting picture quality after decoding, by compressing the reference video frames that are kept in memory during the decoding process.

The paper is divided as follows. Section 2 addresses the most relevant constraints for embedding a compression system in the motioncompensation stage of the MPEG decoder. Section 3 is devoted to the compression systems being used. Section 4 presents simulation results of an experiment where a system was build into a stateof-the-art MPEG-2 decoder. Finally, conclusions are discussed in Section 5.

\section{System requirements}

System costs in the MPEG decoder are dominated by the bi-directional motion compensation unit, which employs two frame memories to store the previous and next reference frames (I and $\mathrm{P}$ pictures). The memory cost of two 4:2:0 sampled images as used in MPEG main level is $10 \mathrm{Mbits}$. The required bandwidth to offer two full-colour reference frames simultaneously is $50-60 \mathrm{MB} / \mathrm{s}$, while the required decoding speed for real-time operation is $16-18 \mathrm{MHz}$. For reduced system costs, the reference frames are typically stored in a Synchronous DRAM (SDRAM) of e.g. 16 Mbits capacity (see Fig. 1(a)). This memory serves also as a background memory for a CPU which controls the MPEG decoder and generates additional OnScreen-Display menus for user control (indicated by the hatched area in Fig. 1(a)). The costs of the memory usage for motion compensation is reduced by recompressing the reference frames prior to memory storage, in order to save memory capacity for other purposes (e.g. CPU data, graphics).

Several important system constraints for the 

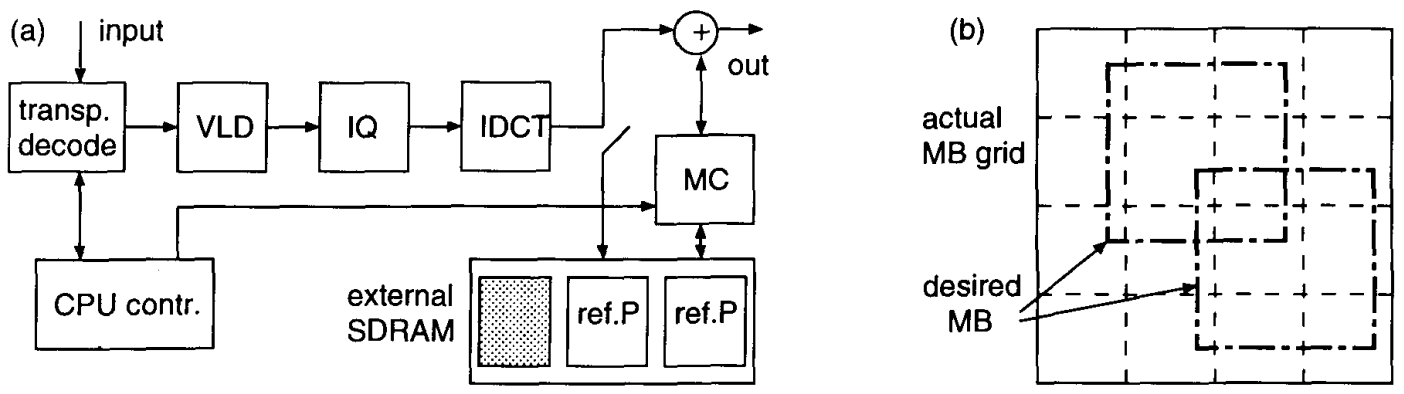

Figure 1: (a) MPEG decoder architecture diagram, (b) Locations of required motion-compensated blocks on actual macroblock (MB) grid.

embedded compression of reference frames in MPEG have to be satisfied and are listed below. The last two are rather special and typical for this type of application.

- High picture quality. The quality of the embedded compression should be well above the MPEG decoder to avoid coding noise interference.

- Low complexity. The complexity should be a fraction of the saved memory costs of the MPEG decoder.

- Accessibility. The motion compensation using a motion vector for each macroblock (MB), requires that MB-data should be retrieved from the background memory on "arbitrary" positions, which mostly do not coincide with the static block grid (see Fig. 1(b)). The embedded compression should allow easy extraction of the reference data.

- Stable quantization after multiple encodings. The recursivity in MPEG, where $\mathrm{P}$-pictures form the input for the subsequent P-picture reconstruction, requires that accurate quantization is performed when embedded compression is inserted. Otherwise, the decoder would drift away from the local reconstruction loop as applied in the encoder, leading to e.g. colour distortion.

\section{Embedded compression system}

Firstly, a framework is presented for two classes of systems, using segmented coding. Secondly, two systems are briefly discussed: block predictive coding and DCT transform coding.

\subsection{Fixed-length segment coding}

To satisfy the accessibility constraint, we use a compression system which generates fixed-length data packets for a group of MPEG macroblocks, called a segment (see Fig. 2). Each group is compressed independently and has a size of 9 MBs. This so-called feedforward coding technique was adopted from digital video recording [2]. The video data is first analysed with a set of various quantization strategies, otherwise fixed-length compression is not guaranteed. The advantage of feedforward coding is that a fixed mapping of the image in the memory is achieved, improving the data accessibility to individual segments without overhead.

It should be noticed that the system in Fig. 2 is very flexible, allowing different implementations and optimizations with respect to a number of system constraints. These constraints are e.g. the desired embedded compression factor, resulting image quality and allowed complexity of the embedded coder. Both sample and frequency-domain coding systems can be employed and can be optimized specifically depending on the chosen architecture. For this reason, we have experimented with a very simple predictive coding scheme and a more expensive transform coding system.

\subsection{Block predictive coding}

A high picture quality and low complexity can be obtained with Block Predictive Coding (BPC) on 


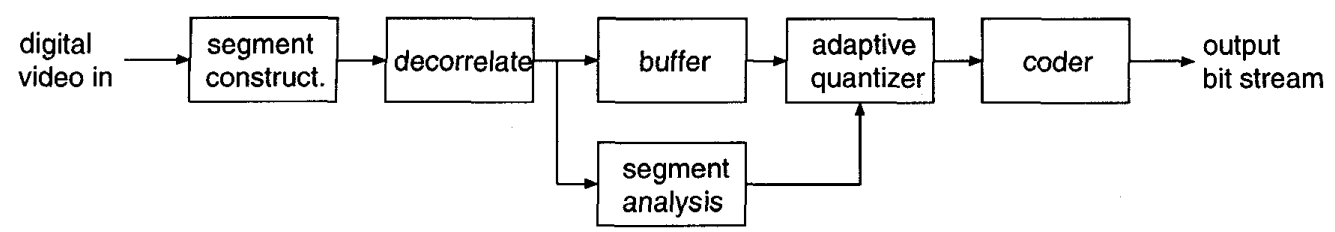

Figure 2: Block diagram of system encoder using feedforward fixed-length compression of segments.

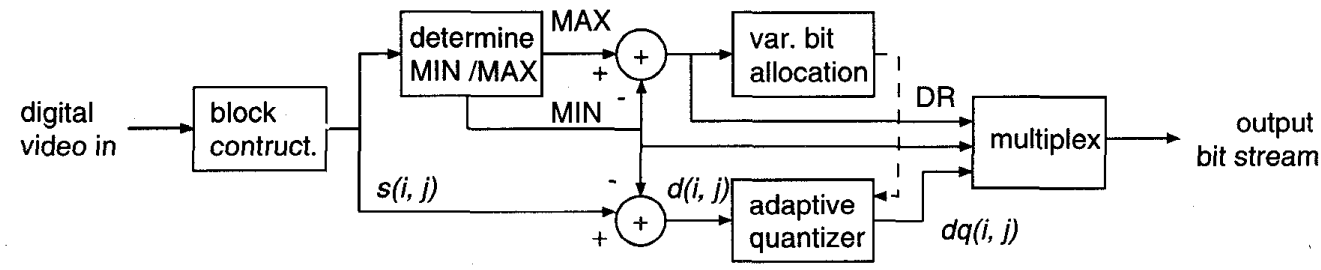

Figure 3: Block diagram of basic BPC system encoder.

a local basis, by using small blocks of $4 \times 4$ pixels. Subsequently, the maximum (MAX) and minimum (MIN) pixel values of each block are determined. The difference between the MAX and MIN value in a block is called Dynamic Range (DR). For low cost, the MIN sample value is transmitted as a global prediction for all other samples inside the block [3] (the decorrelation in Fig. 2). After subtracting this minimum value of the actual sample value $s(i, j)$, all difference values $d(i, j)$ of a block are quantized adaptively, according to the actual block DR, resulting in quantized differences $d q(i, j)$. A block scheme showing the basic steps is portrayed by Fig. 3. For simplicity, quantization based on powers of 2 was implemented. The strategy chosen after segment analysis is the finest quantization that yields the desired number of bits reserved for a group of $9 \mathrm{MBs}$.

\subsection{Transform coding}

A transform coding scheme employing DCT and variable-length coding has also been used for experiments. The DCT transform can be optimized for low-cost hardware [4], and it is inserted in Fig. 2 as the decorrelation step. Furthermore, simple block-based adaptive quantization can be applied. For variable-length coding, joint runlength-amplitude coding, similar to the one used in MPEG [1], has been employed. In this case, accessibility of the data becomes more diffi- cult, since the length of the variable-length codes (VLC) is not known in advance, in contrast with the BPC system (see the next section). This system enables a somewhat larger compression factor (at the expense of increased hardware costs).

\subsection{Accessibility}

In MPEG-2, the motion vector of each macroblock is used to perform the motion compensation. Since this vector may vary from $M B$ to $\mathrm{MB}$, individual macroblocks from the compressed frames have to be recovered.

With the feedforward segment coding (see Section 3.1), resulting in a fixed compression factor, the start position of each segment has a fixed known location in the memory. In this way, fast access to each group of macroblocks is possible. To limit the overhead in memory access to a minimum, all macroblocks should be individually accessible.

In the case of embedded compression using variable-length codes (VLC), each macroblock has a variable bit cost, so that the position of the blocks cannot be easily determined. When using a fixed compression factor per segment (see Section 3.1), only the position of the first macroblock within the segment is known. In the worst case, to access a set of pixels within a segment, the decompression of the complete segment may be required.

However, the attractivity of the BPC system is 


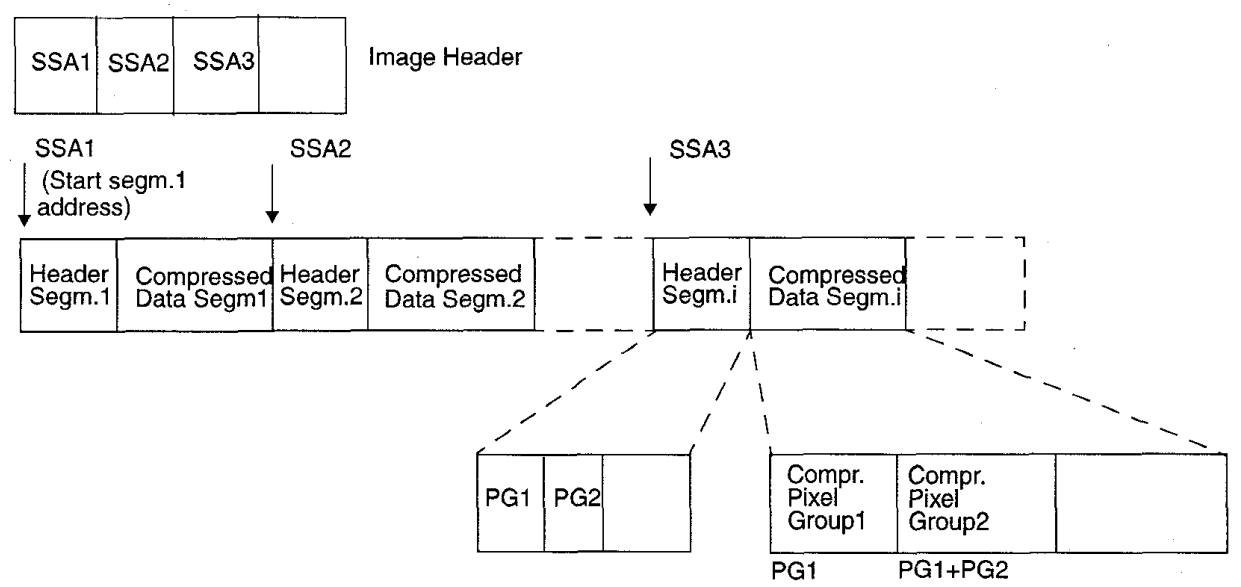

Figure 4: Construction of header to improve data accessibility.

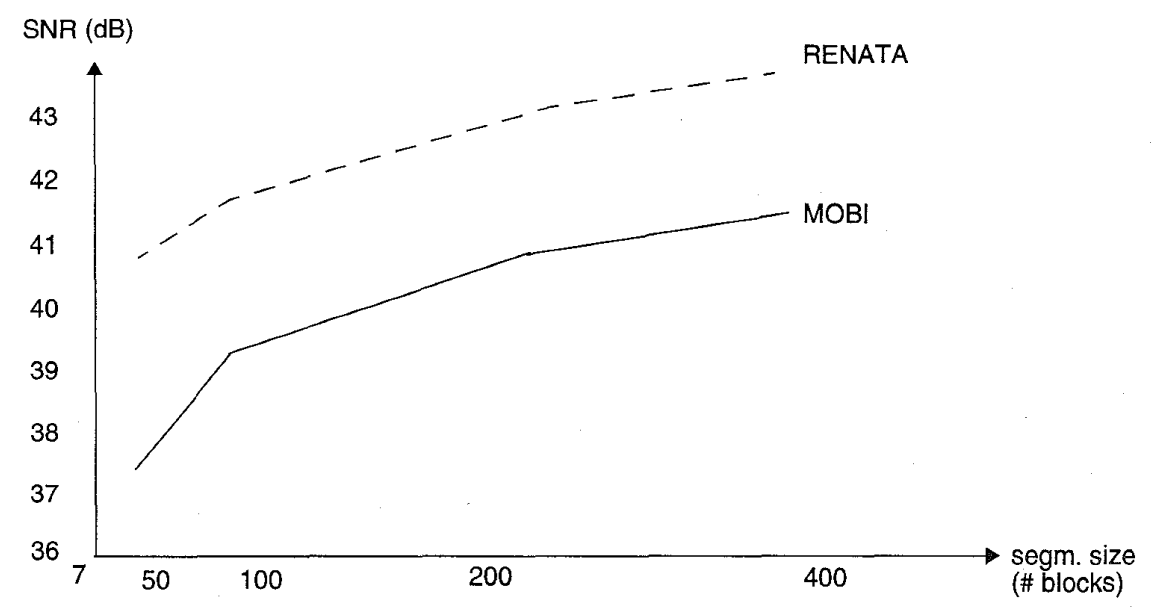

Figure 5: SNR for $\mathrm{Y}$ as a function of the segment size.

that the coding step is integrated into the adaptive quantization stage: the number of bits spent per block are uniquely defined by the combination of the quantizer strategy and the DR parameter of the block. Therefore, the strategy number and the set of DR parameters suffices to compute the starting position of every block in the compressed segment. For this reason, each compressed segment contains a header consisting of the strategy number and DR parameters (see Fig. 4). This header also contains an offset pointer to the start address where of the compressed luminance (Y) blocks are located. In this way, the access to the $\mathrm{Y}$ block is independent of the chrominance $(\mathrm{C})$ blocks. In the segment, the $\mathrm{C}$ blocks are located before the luminance blocks (C needs less bits), leading to a smaller block pointer for the Y blocks. Summarizing, the usage of the described fixedlength segment coding and the efficient header construction lead to attractive properties. Firstly, the memory access map gives marginal overhead (some extra DR parameter reads), while it provides block accessibility. Secondly, a fast access to the different pixels within the image is obtained, without major processing steps in between.

\section{Coding experiments and simu- lations}

The embedded compression system of the previous section has been integrated in the MPEG 


\begin{tabular}{|c|c|c|c|c|}
\hline Image & $\mathrm{CF}=1$ & $\mathrm{CF}=2$ & $\mathrm{CF}=2.3$ & $\mathrm{CF}=2.5$ \\
\hline$\overline{\mathrm{MOBI}}$ & 26.9 & "26.7 & "26.7 & 26.5 \\
\hline Orig. - MPEG & $30.8, \quad 32.7$ & $30.6, \quad 32.5$ & $30.6, \quad 32.4$ & $30.5, \quad 32.3$ \\
\hline $\begin{array}{c}\text { MOBI } \\
\text { MPEG - MPEG Emb. }\end{array}$ & & \begin{tabular}{c}
\multicolumn{2}{c}{45.0} \\
$48.5, \quad 49.0$
\end{tabular} & $\begin{array}{c}41.4 \\
45.6, \quad 46.0\end{array}$ & $\begin{array}{c}38.7 \\
45.6, \quad 46.0\end{array}$ \\
\hline RENATA & 30.9 & 30.8 & 30.6 & 30.5 \\
\hline Orig. - MPEG & $38.2, \quad 37.3$ & $38.1, \quad 37.2$ & $37.9, \quad 37.1$ & 37.9 \\
\hline $\begin{array}{c}\text { RENATA } \\
\text { MPEG - MPEG Emb. }\end{array}$ & & \begin{tabular}{l}
\multicolumn{2}{c}{45.2} \\
$53.0 . \quad 53.2$
\end{tabular} & \begin{tabular}{c}
\multicolumn{2}{c}{45.0} \\
$52.5, \quad 52.6$
\end{tabular} & \begin{tabular}{c}
\multicolumn{2}{c}{44.9} \\
$51.3 . \quad 51.4$
\end{tabular} \\
\hline
\end{tabular}

Table 1: SNR for Y (up) U, V (down) obtained for different compression factors (CF) and segment size $N=216$. In this table, the column with $\mathrm{CF}=1$ means "no embedded compression".

codec designed by the MPEG Software Simulation Group. In order to quantify the loss in image quality from embedded compression, a set of experiments has been conducted. First, the original video image has been compared with the reconstructed MPEG image with and without embedded compression. A set of (embedded) compression factors ranging between $2-2.5$ has been used. The influence of the following parameters has been investigated: quantization strategies, number of blocks within a segment and the chosen compression factors. For the experiments, two sequences were used: a detailed sequence MOBI (coded at $9 \mathrm{Mbit} / \mathrm{s}$ ) and a typical video sequence RENATA (coded at $4 \mathrm{Mbit} / \mathrm{s}$ ).

\subsection{Segment size and picture quality}

In feedforward coding, groups of $N$ blocks are compressed together. It is easily understood that an improved picture quality is obtained for a larger segment size [2], because it leaves more flexibility in bit assignment with respect to detailed and non-detailed areas. However, another tradeoff should be made between image quality and segment memory costs, as this memory is located inside the compression system (see Fig. 2).

The Signal-to-Noise Ratios (SNR), defined as $10 \log _{10}(255)^{2} / \sigma^{2}$ for different segment sizes are depicted in Figure 5. The segment size includes the corresponding colour blocks as well. From Figure 5 , it can be noticed that the SNR increases with the segment size. The increase becomes more smooth at a few hundred data blocks. If a trade- off between segment memory size and image quality should be made, then $N=216$, corresponding with 9 MPEG-2 macroblocks, is a suitable alternative (frame-based processing). A larger number of blocks results in an unnecessary increase of the memory size without significant improvement in SNR. It is emphasized that these results are valid only for a compression factor around 2 .

\subsection{Compression factor and picture quality}

To determine the range of compression factors which can be achieved, employing the described BPC codec, the SNR has been evaluated for different compression factors ranging between 2-2.5 (see Table 1). In the odd rows of the table, the expression "Orig-MPEG" stands for a comparison between the original images and the overall MPEG-decoded quality, including the embedded compression. The even rows of the table refer to a comparison between MPEG decoding and MPEG decoding with embedded compression. The experiments have been performed for a segment size of $N=216$. A comparison between the MPEG2 coder with and without embedded compression, expressed in SNR, is also presented. From Table 1 it becomes clear that, because the SNR obtained with the embedded coder is much higher than that of the MPEG-2 coder, the embedded coder does not interfere with the MPEG-2 decoder. This was also verified with numerous visual experiments. For a higher compression factor, the transform coder can be inserted. 


\subsection{Performance comparison BPC-DCT}

To determine the performance of the DCT-based embedded codec, the SNR has been computed for different compression factors, ranging between 2 and 7. The SNR achieved by the BPC has been already presented in Table 1 for a fixed compression factor of 2-2.5. From the Figure 8, it can be seen that the SNR for an MPEG-2 reconstructed image is much lower than the image quality obtained by both embedded codecs. This large difference in image quality between MPEG-2 and the embedded compression ensures that no visible artifacts occur due to the coding of the reference frames. Figure 8 also shows that the SNR of the blockpredictive codec is somewhat lower than the SNR obtained with the DCT-based codec. This quality difference, valid for a compression factor around 2 , is caused by the more advanced processing in the DCT compression scheme. However, also the hardware requirements are higher for the DCTbased scheme. Therefore, if only a limited compression of the memories is required, employing the block-predictive scheme forms a much better alternative. The relative larger hardware costs required for the DCT-based embedded codec make only compression factors of 3.5 and higher result in an efficient trade-off between the hardware demands for embedded compression and the memory saved.

\subsection{Robustness test: repetitive quan- tization}

In Section 2 it was mentioned that if a long series of consecutive P-pictures is coded without any Iframe in between, a cumulation of errors can occur if the quantization is not dimensioned properly. In our case, we started with using division factors of $2^{n}$, shown in Table 2 . Initially, the quantization matrix from Table 2 was employed, combined with the level-assignment technique 1 from Fig. 7 (left). It can be noticed that the quantization errors are not symmetric about zero, leading to a cumulation of errors for repetitive encoding-decoding loops.

The cumulations of errors can lead to rather annoying artifacts like colour drift. This is shown in Figure 9(left), which portrays the 61 th frame of a GOP, containing one I-picture followed by $60 \mathrm{P}$ -

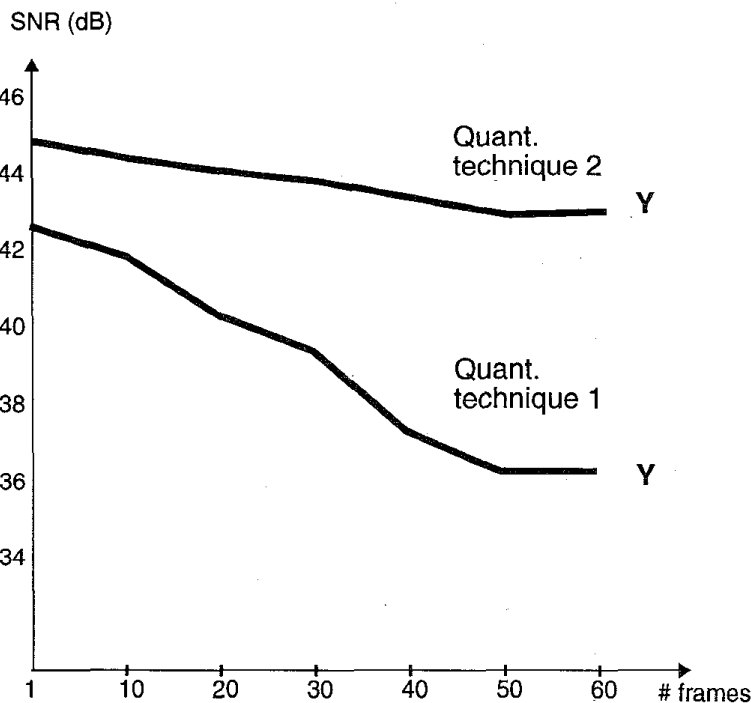

Figure 6: SNR for $\mathrm{Y}$ as a function of the frame number in a long GOP, so that repetitive quantization is used.

pictures in which embedded compression has been employed. In order to create robustness for repetitive coding, other quantization techniques should be adopted.

Quantization schemes exist where the number of positive and negative error offsets are equal, thereby avoiding the creation of a DC-offset. An example of a quantizer level assignment without DC-errors (quantization technique 2), is depicted in Figure 7 (right). This technique represents a dead-zone quantizer, in which the number of values that introduce a negative error offset equals the number of values with a positive error offset. Clearly, such a level assignment is robust for multiple encoding-decoding loops. The 61th frame of the same GOP of 61 pictures with embedded compression using the second quantization technique is portrayed in Figure 9 (right). As we can see, the cumulation of errors does no longer occur. In Figure 6, the SNR for the two quantization techniques after the 1st and 61th P-frames are depicted.

\subsection{Enhanced compression by VLC}

To enhance the compression factor of the BPC system, additional variable-length coding (VLC) of the quantized difference samples has been in- 


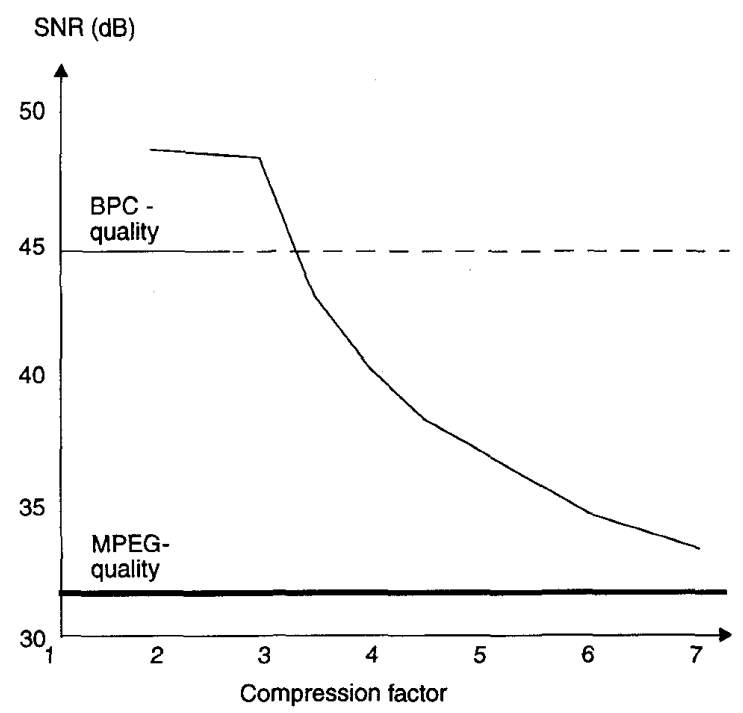

Figure 8: Compression performance of DCTbased codec for the sequence "MOBI".

vestigated. Firstly, the statistics of the video data after BPC were analyzed [7], leading to the following conclusions.

- The distribution of video data after decorrelation and quantization is almost stationary and decays exponentially with the amplitude of the quantized video signal (adding VLC would be beneficial);

- The measure of activity within the block (dynamic range) inside a small block of video data is a key parameter for the variable length code generation.

- The quantization strategy (i.e. coarseness) selected does not influence the statistics of the YUV-component signal.

Based on these observations, experiments were performed with Huffman and arithmetic coding, both for adaptive and non-adaptive coding. For non-adaptive coding, both Huffman and arithmetic coding achieve an improvement of the compression performance with almost $10 \%$. If the algorithms employ fixed VLC tables which are adaptive to the dynamic range, the efficiency improvement is roughly doubled to approximatively $20 \%$. An extra improvement of $5 \%$ is obtained with an arithmetic coder that is adaptive to the occurrence of symbols within a specific DR class. If a trade-off between the complexity and the performances of the different entropy coders need to be made, then the Huffman code adaptive to the Dynamic Range forms the best proposal.

\subsection{Accessibility of BPC with VLC}

If the compression system is extended with VLC, the starting positions of the $4 \times 4$ sample blocks can no longer be determined easily, because a unique relation between the (Dynamic Range, Strategy) and the bit-cost per sample ceases to exist. If entropy coding is used, the length of the coded pixels are represented by a variable number of bits. In order to provide individual access to each block, so-called entry points, representing the start address of the $4 \times 4$ sample blocks, have to be stored in a header. The position of each block is coded as an offset to the start address of the previous block. If the maximum number of bits required for the coded representation of a signal sample (i.e. largest Huffman code) equals 16 bits, then the maximum total number of bits required for the coded representation of a block is less than 256. This implies that 8 bits are sufficient to represent the offset to the start position of the next block.

The potential gain of an additional $20 \%$ (about 12 bits per block, compr. factor 2) resulting from adaptive Huffman coding, is not realized, since block access ( 8 bits) reduces the gain to $6 \%$. This can be improved by relaxing the desire to access each individual block, such that access to every two blocks is possible (range max. 512 bits $=9$ bit offset address). In the latter case, the compression improvement from adaptive Huffman coding is about $13 \%$. Clearly, the trade-off to be made is between improved compression, costs of adding VLC and fast accessibility of individual pixels.

\section{Conclusions}

A simple low-cost compression system has been added to the motion-compensation loop of the MPEG-2 decoder, to reduce its memory cost. It 


\begin{tabular}{|c|c|c|c|c|c|c|c|c|}
\multicolumn{10}{c|}{ Strategy number } \\
\hline \hline Dynamic Range & 0 & 1 & 2 & 3 & 4 & 5 & 6 & 7 \\
\hline \hline$-1 . .0$ & 1 & 2 & 2 & 2 & 2 & 2 & 2 & 2 \\
\hline$-2 . .1$ & 1 & 2 & 2 & 4 & 4 & 4 & 4 & 4 \\
\hline$-4 . .3$ & 1 & 2 & 2 & 4 & 4 & 4 & 4 & 4 \\
\hline$-8 . .7$ & 1 & 2 & 2 & 4 & 8 & 8 & 8 & 8 \\
\hline$-16 . .15$ & 1 & 2 & 4 & 4 & 8 & 8 & 8 & 8 \\
\hline$-32 . .31$ & 1 & 2 & 4 & 4 & 8 & 8 & 8 & 8 \\
\hline$-64 . .63$ & 1 & 2 & 4 & 4 & 8 & 8 & 16 & 16 \\
\hline$-128 . .127$ & 1 & 2 & 4 & 4 & 8 & 16 & 16 & 32 \\
\hline \hline
\end{tabular}

Table 2: Quantization step size as function of the strategy and the dynamic range.
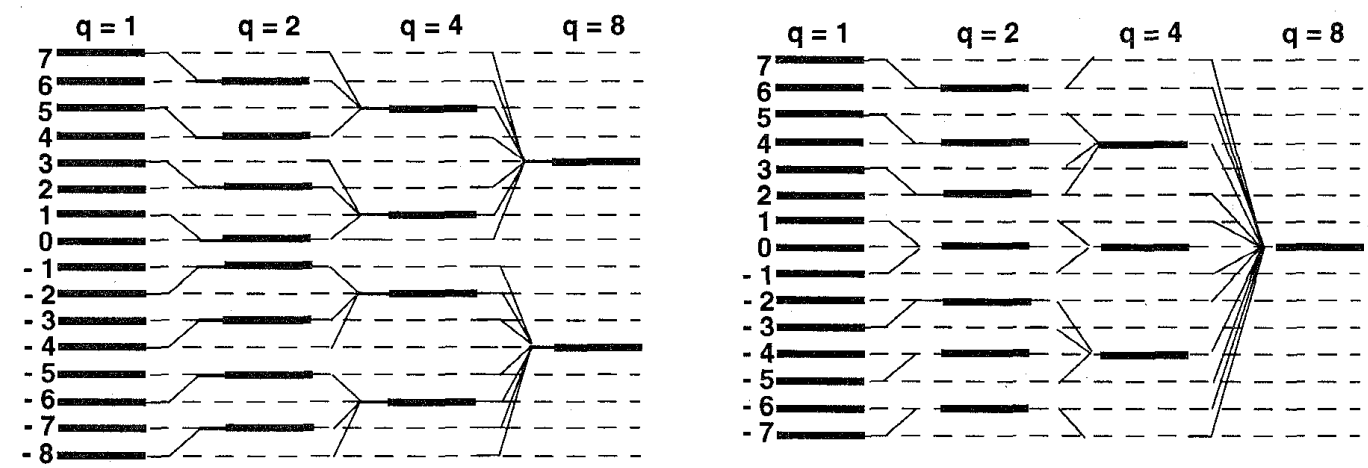

Figure 7: Quantization technique 1 (left), not robust, and 2 (right) which is robust for repetitive coding.

was shown that the picture quality of the reconstructed MPEG image sequence (MOBI, SNR 26$30 \mathrm{~dB}$ ) is hardly influenced by the embedded compression which operates at a much higher quality (SNR 39-45 dB). The negligible loss of embedded compression is substantiated by simulation measurements (Table 1) and is confirmed in other publications [5] [6]. For lower bit rates, (e.g. $4 \mathrm{Mbit} / \mathrm{s}$ ), the SNR between the original and the reconstructed MPEG-2 image decreases, such that the losses introduced by embedded compression are even vanishing, permitting the embedded codecs to achieve either higher compression factors or reduced system costs.

It was shown that careful quantizer design is required, to maintain a stable SNR after multiple encodings occurring in large GOP sizes in MPEG. Quantizers should have no DC offset in order to keep the SNR loss within $1 \mathrm{~dB}$.

In an earlier paper [7], improved compression results have been presented by adding variable- length coding (arithmetic coding) to the BPC system. However, due to the unknown variable number of bits per quantized data block, the accessibility scheme becomes more difficult and expensive in overhead. As a consequence, the number of bits gained, are partially lost for creating sufficient accessibility of individual data blocks.

Since the proposed technique considers the reference pictures in the memory as normal images, it may be equally applied in both MPEG-2 encoders and in MPEG-2 decoders. This transparency is not so obvious in alternative proposals for embedded compression in MPEG. The simplicity of the proposed feedforward BPC system, together with the promising simulation results, have resulted in the decision to include the system into a commercial IC design for further testing and evaluation. 

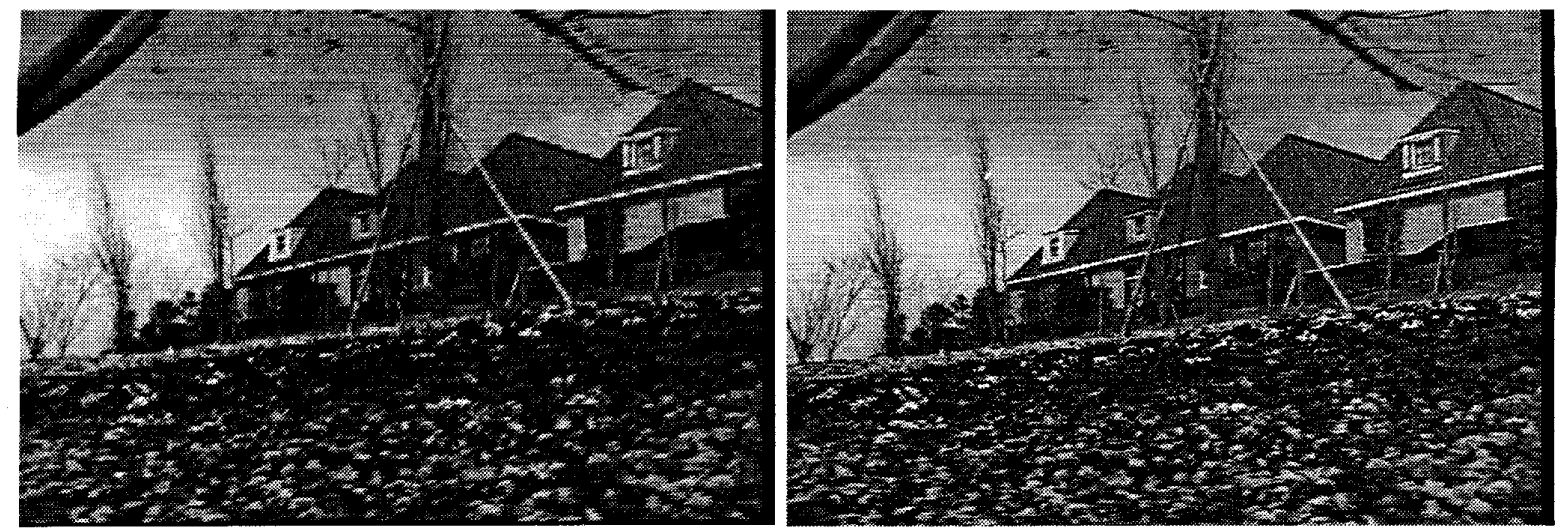

Figure 9: Illustration of the color-drift due to the cumulation of errors. Reconstructed images with quantization technique 1 (left) and 2 (right).

\section{References}

[1] "Coding of Moving Pictures and Associated Audio", International Standard, ISO/IEC 13818, November 1994.

[2] P.H.N. de With and A.M.A. Rijckaert, "Design considerations of the video compression system of the new digital DV recording standard", IEEE Trans. Consum. Electron., Vol. 43, No. 4, pp. 1160-1179, November 1997.

[3] T. Kondo et.al., "A new ADRC for consumer digital VCR", IEE Proc. 8th VADR Conf., Birmingham (UK), IEE Publ. No. 319, pp. 144-150, April 1990.

[4] M. v.d. Schaar-Mitrea and P.H.N. de With, "On the application of fast DCT transforms for combined SW/HW implementation", Proc. 18th Symp. on Inform. Theory in the Benelux, Veldhoven, The Netherlands, pp 33-40, May 1997.

[5] D. Pau and R. Bruni, "A novel adaptive vector quantization method for memory reduction in MPEG-2 HDTV decoders", IEEE Trans. Consum. Electron., Vol. 44, August 1998, to appear.

[6] R.P. Kleihorst and F. Cabrera, "Motion estimation and compensation in the DCT-domain with implementational aspects", Proc. 19th Symp. on Information Theory in the Benelux, Veldhoven, The Netherlands, May 1998.

[7] M. v.d. Schaar-Mitrea and P.H.N. de With, "A comparison between Huffman and arithmetic coding for video compression", Proc. 17th Symp. on Inform. Theory in the Benelux, Enschede, The Netherlands, pp 25-30, May 1996.

Peter H.N. de With was born in Lexmond, The Netherlands, in 1958. He graduated in electrical engineering from the University of Technology in Eindhoven. In 1992 he received the Ph.D. degree from the University of Technology Delft, The Netherlands, for his work on video bit-rate reduction for recording applications. He joined Philips Research Laboratories Eindhoven in 1984, where he became a member of the Magnetic Recording Systems Department. From 1985 to 1993 he was involved in several European RACE projects (a.o. 1001 and 2026) in which digital SDTV, HDTV and data recording was studied. In the early nineties he contributed as a video coding expert to the DV standardization committee. Since 1994 he became a member of the TV System group where he is working on advanced video processing architectures for various enhancements and compression systems. In 1996 he became senior TV systems architect and in October 1997 he was appointed as full professor at the University of Mannheim, Germany. Regularly, he is a teacher of the Philips Centre for Technical Training and for other public post-academic courses on digital video compression and recording and video processing 

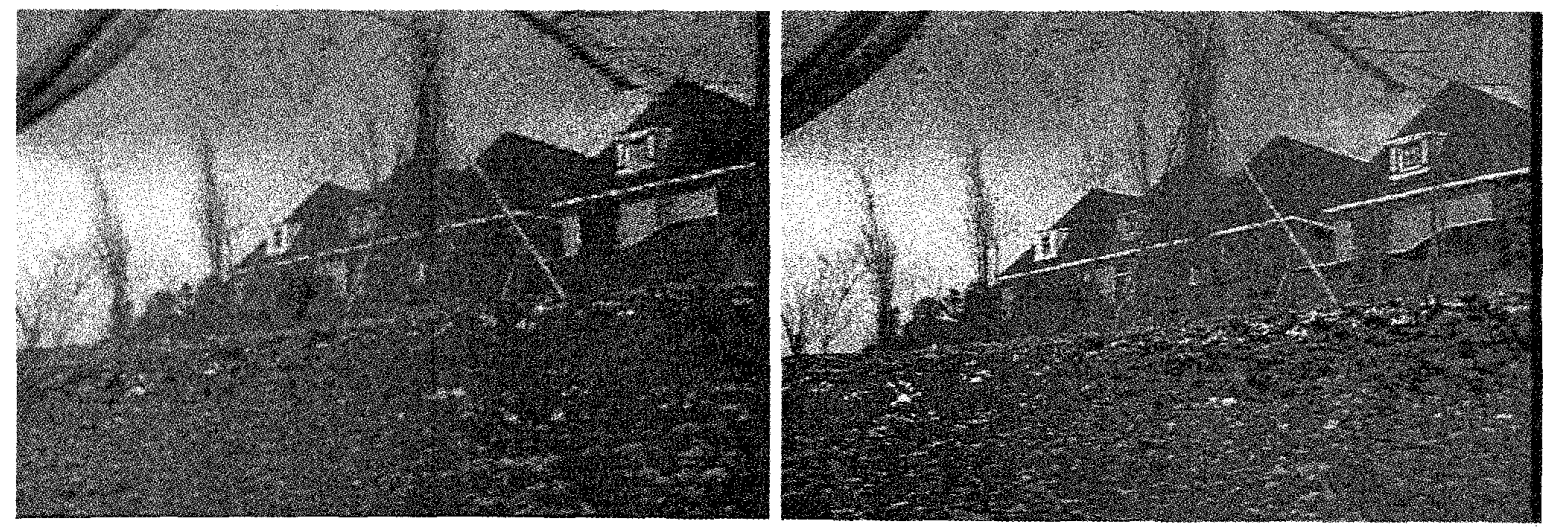

Figure 9: Illustration of the color-drift due to the cumulation of errors. Reconstructed images with quantization technique 1 (left) and 2 (right).

\section{References}

[1] "Coding of Moving Pictures and Associated Audio", International Standard, ISO/IEC 13818, November 1994.

[2] P.H.N. de With and A.M.A. Rijckaert, "Design considerations of the video compression system of the new digital DV recording standard", IEEE Trans. Consum. Electron., Vol. 43, No. 4, pp. 1160-1179, November 1997.

[3] T. Kondo et.al., "A new ADRC for consumer digital VCR", IEE Proc. 8th VADR Conf., Birmingham (UK), IEE Publ. No. 319, pp. 144-150, April 1990.

[4] M. v.d. Schaar-Mitrea and P.H.N. de With, "On the application of fast DCT transforms for combined SW/HW implementation", Proc. 18th Symp. on Inform. Theory in the Benelux, Veldhoven, The Netherlands, pp 33-40, May 1997.

[5] D. Pau and R. Bruni, "A novel adaptive vector quantization method for memory reduction in MPEG-2 HDTV decoders", IEEE Trans. Consum. Electron., Vol. 44, August 1998, to appear.

[6] R.P. Kleihorst and F. Cabrera, "Motion estimation and compensation in the DCT-domain with implementational aspects", Proc. 19th Symp. on Information Theory in the Benelux, Veldhoven, The Netherlands, May 1998.

[7] M. v.d. Schaar-Mitrea and P.H.N. de With, "A comparison between Huffman and arithmetic coding for video compression", Proc. 17th Symp. on Inform. Theory in the Benelux, Enschede, The Netherlands, pp 25-30, May 1996.

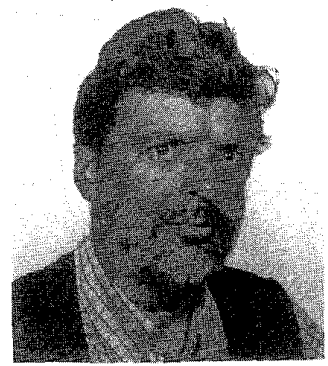

Peter H.N. de With was borm in Lexmond, The Netherlands, in 1958. He graduated in electrical engineering from the University of Technology in Eindhoven. In 1992 he received the Ph.D. degree from the University of Technology Delft, The Netherlands, for his work on video bit-rate reduction for recording applications. He joined Philips Research Laboratories Eindhoven in 1984, where he became a member of the Magnetic Recording Systems Department. From 1985 to 1993 he was involved in several European RACE projects (a.o. 1001 and 2026) in which digital SDTV, HDTV and data recording was studied. In the early nineties he contributed as a video coding expert to the DV standardization committee. Since 1994 he became a member of the TV System group where he is working on advanced video processing architectures for various enhancements and compression systems. In 1996 he became senior TV systems architect and in October 1997 he was appointed as full professor at the University of Mannheim, Germany. Regularly, he is a teacher of the Philips Centre for Technical Training and for other public post-academic courses on digital video compression and recording and video processing 
issues. In 1995 he co-authored the paper that received the IEEE CES Transactions Paper award. In 1996, he received a company Invention Award. Mr. de With is a senior member of the IEEE, and board member of the IEEE Benelux Chapter on Consumer Electronics and the Benelux working group for Information and Communication theory.

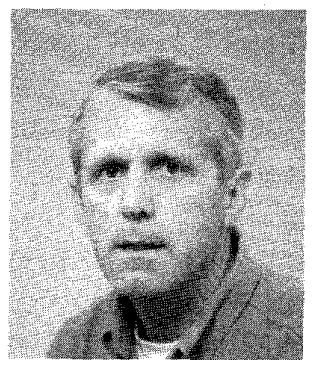

Peter H. Frencken was born in Weert, the Netherlands in 1954. He graduated in electrical engineering from the Polytechnic College in Eindhoven in 1973. He joined Philips Research laboratories the same year where he worked on various subjects related to digital video processing. In 1984 he transferred to the system laboratory of Philips Semiconductors in Eindhoven where he was engaged in subjects related to feature TV and HDMAC. Since 1992 he is involved in the system architecture for MPEG based systems.

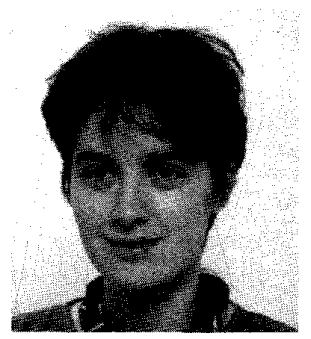

Mihaela van der Schaar-Mitrea was born in Bucharest, Romania, in October 1971. She graduated in electrical engineering (Ir. degree) from the Eindhoven University of Technology in The Netherlands in 1996. She joined Philips Research Laboratories Eindhoven in the same year where she became a member of the TV Systems Department. She is currently involved in the research of video-data compression techniques and their implementation for TV and computer systems. Her research interests include also video and graphics coding and image analysis and she co-authored a series of papers in this field. 